\title{
Reply to Editorial: Interpretation of OCT and fundus findings in COVID-19 patients in recent Lancet publication
}

\author{
Paula M. Marinho $\mathbb{1}^{1,2} \cdot$ Heloisa Nascimento ${ }^{1,2} \cdot$ Allexya A. A. Marcos ${ }^{1,2} \cdot$ André C. Romano $^{1,2} \cdot$ Richard B. Rosen ${ }^{3} \cdot$ \\ Rubens Belfort Jr ${ }^{1,2}$
}

Received: 28 July 2020 / Revised: 5 October 2020 / Accepted: 2 November 2020 / Published online: 25 November 2020

(c) The Royal College of Ophthalmologists 2020

\section{To the Editor:}

We have read the Editorial [1] regarding our publication [2] and would kindly request to respond in order to clarify our original observations.

Our patients' age ranged from 25 to 69 years old (mean: 43 years; median: 40 years). Of the 12 patients from our original report, seven had no comorbidities, one had controlled type 2 diabetes and high blood pressure (HBP), two had HBP with good clinical control, one had a history of dyslipidemia, and one had ankylosing spondylitis, controlled with adalimumab, and no history of ocular involvement.

We do agree that cotton wool spots (CWS) can be identified in a broad spectrum of diseases. On Fig. 1 we present a follow-up composite of the patient presented at the Lancet Publication showing regression of the cited lesion. The CWS image was not a NFL myelination and disappeared as expected.

At present moment we have seen close to 200 patients, ranging from asymptomatic to deceased after intensive care unit admission. To better illustrate our findings, we present images from a 52-year-old female, who was nasal swab positive for SarS-CoV-2, hospitalized for hypoxia, displaying subtle CWS in the right eye (OD) and no alterations in the left eye. Figure 2 is a composite of her color fundus photograph and OD B-scan OCT, which demonstrates a

Heloisa Nascimento

helomn@gmail.com

1 Instituto Paulista de Estudos e Pesquisas em Oftalmologia IPEPO/Instituto da Visão, São Paulo, Brazil

2 Ophthalmology Department, Federal University of Sao Paulo, Hospital São Paulo, São Paulo, Brazil

3 New York Eye and Ear Infirmary of Mount Sinai, New York, NY, USA hyperreflective lesion, deep to the retinal nerve fiber layer, at the level of the inner plexiform and ganglion cell layer. The red line points to its appearance on the enface OCT image. The OCT-angiogram reveals a flow deficit at the same site. The red dot flow overlay on the B scan OCT image, based upon OCT angiogram data, differentiates this lesion and an adjacent blood vessel cross-section by the presence of the red within the vessel lumen indicating active blood flow. The density of the vessel results in a shadow projecting deep into the underlying retinal layers while the lesion in question does not. The other eye was normal. On Fig. 3 we can see a follow-up composite of the same patient, where although the lesion is no longer visible on the fundus exam we can still spot it on b-scan OCT.

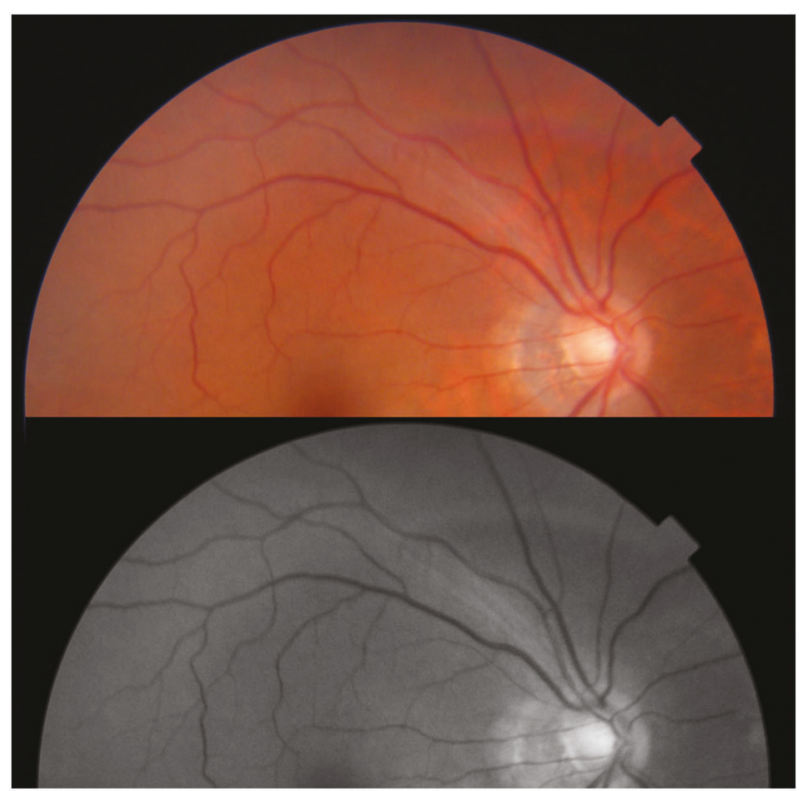

Fig. 1 Fundus picture from right eye of previously published patient (Marinho et al. [1]). The pictures were taken 3 months after first evaluation and shows the absence of the previously reported cotton wool spot lesion. 
Fig. 2 Composite with color fundus photograph (a) and a B-scan OCT (e) of the right eye of patient with preovius COVID-19 diagnosis. The images depict a hyperreflective lesion at the level of the inner plexiform and ganglion cell layer (yellow arrows), which aligns with inferior lesion at the enface OCT image as

highlighted by in red line (c, d, e). The OCT-angiography indicates no flow void at that same site (b). The red dot flow overlay on the B scan OCT image, based upon OCT angiogram data, differentiates this lesion (green arrow) and from an adjacent blood vessel cross-section by the presence of the red overlay within the lumen marking active blood flow (blue arrow). The density within the vessel results in a shadow projected deep into the underlying retinal layers (d), which the lesion produces no shadow artifact.
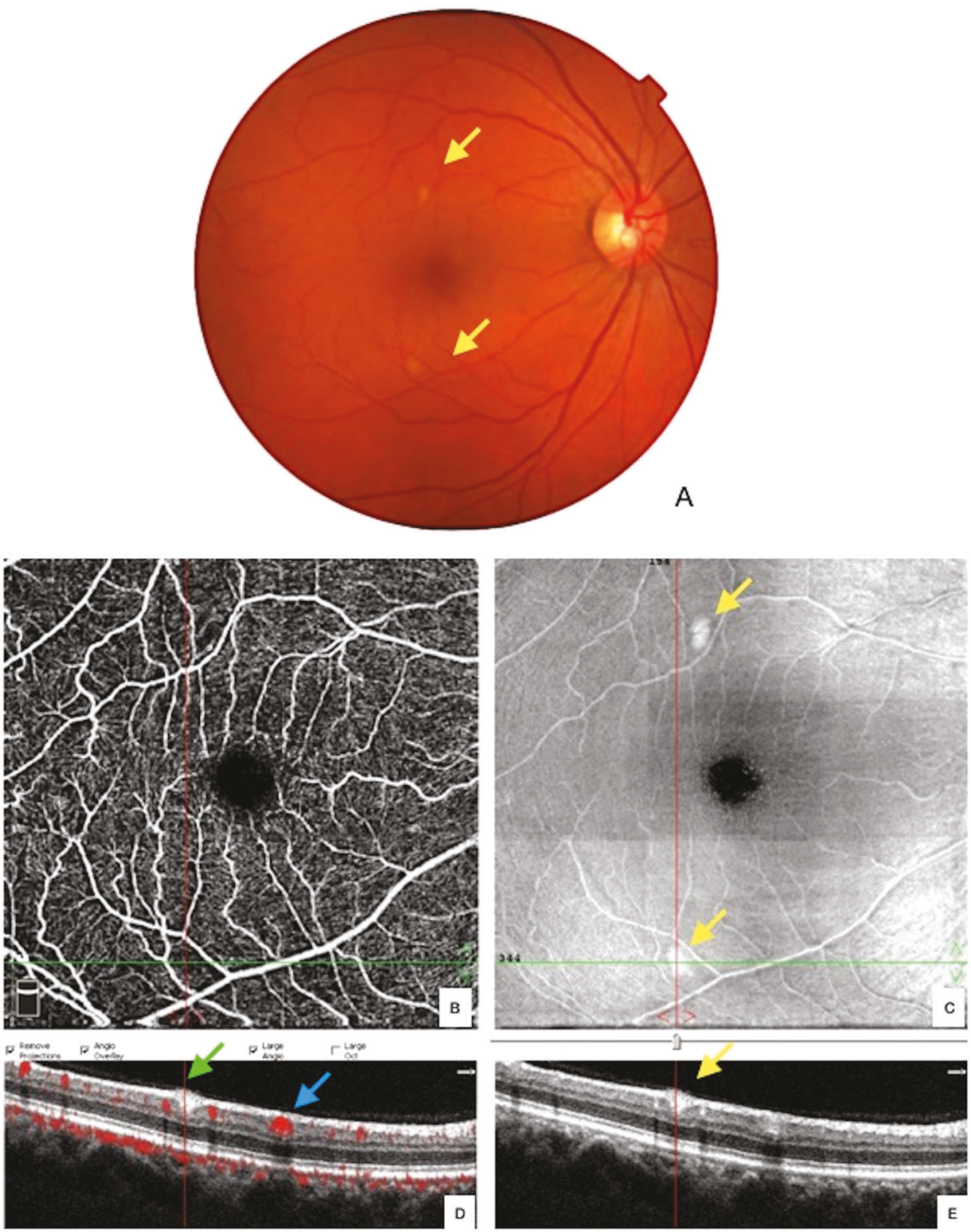


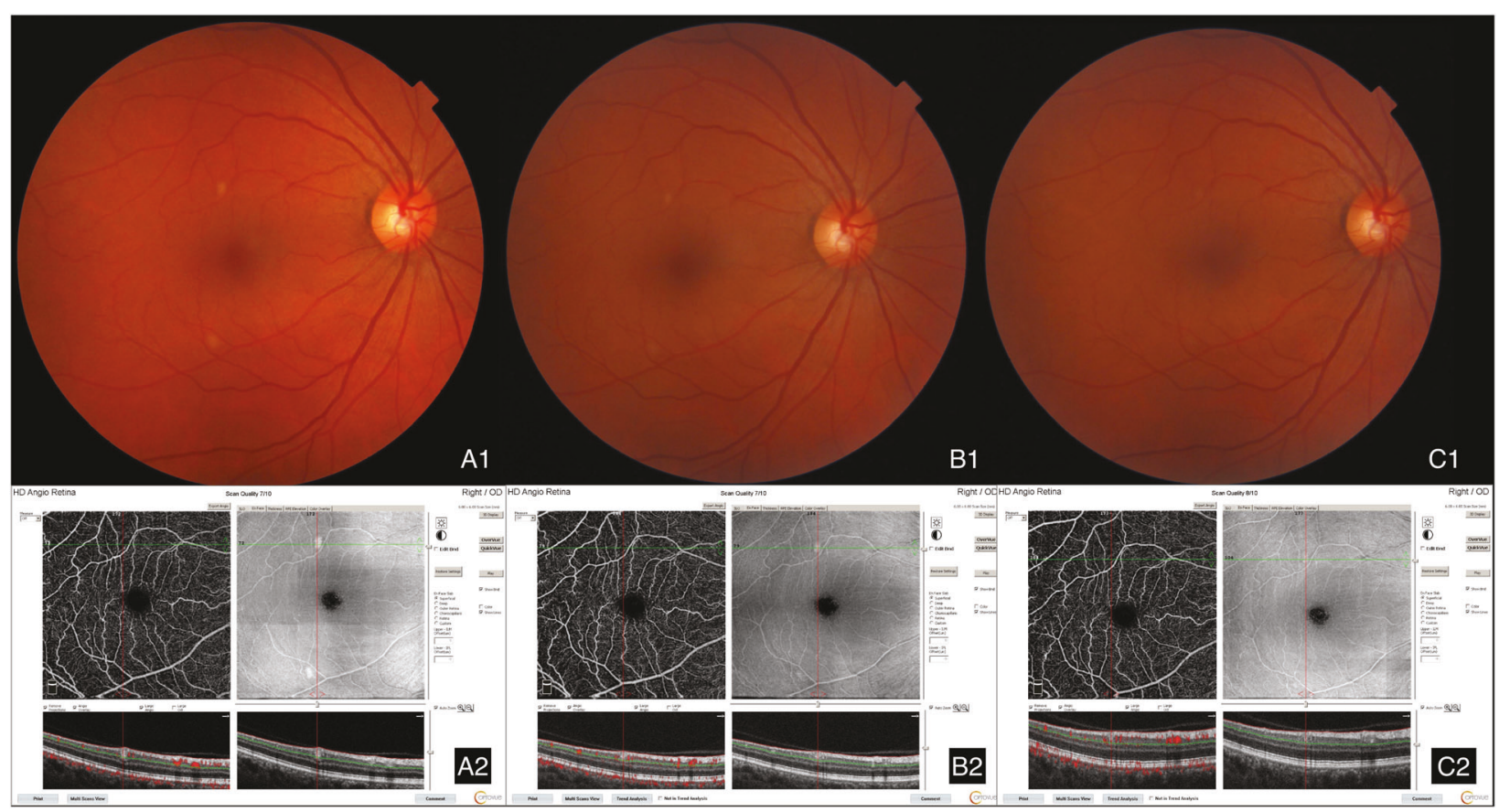

Fig. 3 A composite with fundus exam and OCT from the same patient from Fig. 2 showing lesion's evolution. a1 and a2 were taken on 1 st evaluation, $\mathbf{b} 1$ and $\mathbf{b} 2$ were taken after 37 days. $\mathbf{c} 1$ and $\mathbf{c} 2$ were taken after another 28 days.

Our findings are consistent with other reports that are now been presented [3-5]. We do appreciate all the input received. More data regarding our studies will be promptly presented.

\section{Compliance with ethical standards}

Conflict of interest The authors declare that they have no conflict of interest.

Publisher's note Springer Nature remains neutral with regard to jurisdictional claims in published maps and institutional affiliations.

\section{References}

1. Marinho PM, Marcos AAA, Romano AC, Nascimento H, Belfort R. Retinal findings in patients with COVID-19. Lancet. 2020. https://doi.org/10.1016/S0140-6736(20)31014-X.
2. Vavvas DG, Sarraf D, Sadda SR. Concerns about the interpretation of OCT and fundus findings in COVID-19 patients in recent Lancet publication. Eye (Lond). 2020;9:1-2.

3. Virgo J, Mohamed M. Paracentral acute middle maculopathy and acute macular neuroretinopathy following SARS-CoV-2 infection. Eye. 2020. https://doi.org/10.1038/s41433-020-1069-8.

4. Landecho MF, Yuste JR, Gándara E, Sunsundegui P, Alcaide AB, García-Layana A. COVID-19 retinal microangiopathy as an in vivo biomarker of systemic vascular disease? J Intern Med. 2020. https:// doi.org/10.1111/joim.13156.

5. Insausti-García A, Reche-Sainz JA, Ruiz-Arranz C, López Vázquez Á, Ferro-Osuna M. Papillophlebitis in a COVID-19 patient: Inflammation and hypercoagulable state. Eur J Ophthalmol. 2020:1120672120947591. https://doi.org/10.1177/1120672120947591. 